\title{
Phosphor-Concentration-Dependent Characteristics of White LEDs in Different Current Regulation Modes
}

\author{
JIUN PYNG YOU, ${ }^{1}$ NGUYEN T. TRAN,${ }^{1}$ YUAN-CHANG LIN,${ }^{1}$ YONGZHI \\ $\mathrm{HE},{ }^{1}$ and FRANK G. SHI ${ }^{1,2}$ \\ 1.-Optoelectronics Packaging and Materials Labs, University of California, 916 Engineering
}

Tower Irvine, Irvine, CA 92697, USA. 2.—e-mail: fgshi@uci.edu

\begin{abstract}
Phosphor-concentration-dependent characteristics of white light-emitting diodes (LEDs) under different current regulation conditions were investigated. It is found that the phosphor conversion efficiency of white LEDs driven under constant current is lower than that under pulse current. In addition, white LEDs driven under constant current exhibit higher junction temperature than under pulse current, and the difference is phosphor concentration dependent. For both pulse and constant current modes, white LEDs show relatively stable optical characteristics at relatively higher drive currents, when relatively higher phosphor concentrations are used. At relatively higher phosphor concentrations, the correlated color temperature and the chromaticity coordinates have also been observed to be relatively stable for white LEDs in both constant and pulse current modes.
\end{abstract}

Key words: Light-emitting diodes, phosphor, current regulation, junction temperature

\section{INTRODUCTION}

Continuous improvements a in the lifetime, stability, and light conversion efficiency of light-emitting diodes (LEDs) are urgently needed for LED devices being adopted as next-generation lighting sources, because of their intrinsic high energy efficiency, lack of pollution, high quality, as well as compact size. Moreover, one of the important advantages of LED devices is their capability to adjust the optical output by directly regulating the input current without sophisticated electronics devices. Nevertheless, stable optical output characteristics of white LEDs are necessary for operation under different current regulation conditions. Different current regulation modes have recently been investigated to understand their impacts on junction temperature and thus the lifetime of LEDs. ${ }^{1}$ It is found that the rise of junction temperature in the pulse current mode is limited in comparison with the constant current mode. ${ }^{2}$ With the increase of

(Received July 31, 2008; accepted March 3, 2009;

published online April 3, 2009) pulse width, the junction temperature increases and finally coincides with the value in the constant current mode. ${ }^{3}$ Moreover, the junction temperature difference is enhanced by increasing the overflow of injection current. The high possibility of nonradiative recombination of charge carriers with increased junction heat leads to the reduction of light output of LEDs. ${ }^{4}$

In typical phosphor-converted white LED packages, heat is generated and transferred to the phosphor coating layer with increasing LED input power. However, the phosphor materials used in white LED packages are thermally sensitive. Overheating of the phosphor layer can lower the light conversion efficiency and shift the chromaticity of LEDs. ${ }^{5}$ Moreover, the phosphor concentration and configuration also shift light-emitting characteristic of white LEDs. ${ }^{6}$ Studies have shown that phosphor-converted white LEDs have a relatively small chromaticity shift compared with red-greenblue (RGB) mixed-color white LEDs under different dimming schemes. ${ }^{7}$ In addition, the chromaticity shift of white LEDs under pulse-width modulation is less than that under constant current. ${ }^{8}$ However, 
there is no report on how junction temperatures and light-emitting characteristics of white LED devices vary with phosphor concentration under different current regulation conditions. In this report, the junction temperature is referred to the temperature of the LED chip, specifically the temperature of the $p-n$ junction of an LED chip. By comparing the experimental results of LED devices in pulse and constant current modes, the effects of junction heat and phosphor concentration on light-emitting characteristics can be separated.

This study has demonstrated that, with relatively higher phosphor concentration, optical characteristics of phosphor-converted white LEDs under current regulation conditions are more stable, i.e., the shift of the intensity ratio of blue to yellow band is smaller. In addition, the junction temperatures of white LEDs increase with the increase of phosphor concentration under constant current operation. These results are valuable to understand the capability of phosphor-converted white LEDs in current regulation applications.

\section{EXPERIMENTAL}

In this experiment, GaN-based blue power LED chips made by SemiLEDs Inc. were used. The chip is fabricated with thinned $n$-GaN up structure to enhance light output efficiency and maximize the vertical injection of current into the active layer. The chip has a total thickness of $145 \mu \mathrm{m}$ and its active layer is located at $140 \mu \mathrm{m}$ from the base. The LED chip has a light-emitting area of $1 \mathrm{~mm}^{2}$ and a peak wavelength of $460 \mathrm{~nm}$. The typical forward current is $350 \mathrm{~mA}$ and the recommended maximum forward current should not exceed $600 \mathrm{~mA}$. The leadframes are designed with 1-mm-thick copper substrate coated by $2-\mu \mathrm{m}$ silver. The LED packages are encapsulated with mixtures of YAG:Ce phosphor and silicone, which have a phosphor concentration of $4 \mathrm{wt} . \%, 8 \mathrm{wt} . \%$, and $13 \mathrm{wt} . \%$, respectively. Adjustable pulse current and constant direct current (DC) sources with corresponding indicated forward voltage were supplied by a power generator from Everfine Co., Ltd. Each LED device was driven at six current conditions, i.e., $50 \mathrm{~mA}, 100 \mathrm{~mA}$, $200 \mathrm{~mA}, 350 \mathrm{~mA}, 500 \mathrm{~mA}$, and $600 \mathrm{~mA}$ in pulse and constant current modes. The corresponding lightemitting characteristics of LEDs in terms of light output, correlated color temperature (CCT), and chromaticity coordinates were measured by a spectral light measurement system from LabSphere Inc. In the pulse current operation of LEDs, the pulse width was set at $30 \mathrm{~ms}$ in order to fit the saturation time of light output measurements, so that the input power of the pulse current did not affect the junction temperature significantly. ${ }^{2}$ Therefore, the junction temperatures of LEDs under pulse current operation are basically the same as ambient temperature. On the other hand, for LEDs under constant current operation, a DC was supplied.
The junction temperatures of LEDs increased and finally reached thermal equilibrium with ambiance. The corresponding junction temperatures of LEDs were determined by the forward voltage method in constant current mode. ${ }^{9}$ The diode forward voltage method consists of two series of measurements, a calibration measurement and a real junction temperature measurement. In the calibration measurement, the LED is placed in a temperaturecontrolled oven and connected to the drive and measurement equipment. After the junction has come to thermal equilibrium with the temperaturecontrolled oven, a pulse current with a duty cycle of $0.1 \%$ is sourced into the LED to ensure the junction temperature is equal to the oven temperature, and the voltage drop is measured. In real application, a linear expression is fitted with the corresponding voltage drop with various set points of oven temperature in order to get a calibration curve. The calibration curve serves as the reference for the deduction of the junction temperature from DC measurement and establishes the relation between the forward voltage and the junction temperature.

\section{RESULTS AND DISCUSSION}

A schematic diagram of the high-power LED package is shown in Fig. 1. This phosphor-based white LED package has a uniform phosphor distribution in a silicone encapsulant. The phosphor concentration is controlled to generate different proportions of blue and yellow light such that the chromaticity of the resulting white light is close to the Planckian locus. Figure $2 \mathrm{a}-\mathrm{c}$ shows the emission spectra of LEDs with phosphor concentrations of $4 \mathrm{wt} . \%, 8 \mathrm{wt} . \%$, and $13 \mathrm{wt} . \%$, respectively, driven at $350 \mathrm{~mA}$ under pulse and constant current. For phosphor-converted white LEDs, the emission spectra consist of two distinct emission bands clearly resolved at $460 \mathrm{~nm}$ emitted from LED chips and at around $555 \mathrm{~nm}$, which is emitted by phosphor following the absorption of blue light. As the phosphor concentration increases, the blue spectrum decreases drastically whereas the yellow spectrum becomes saturated in the dichromatic configuration. In addition, the emission power of LEDs in a constant current mode is rather smaller compared with in a pulse current mode. Figure 3

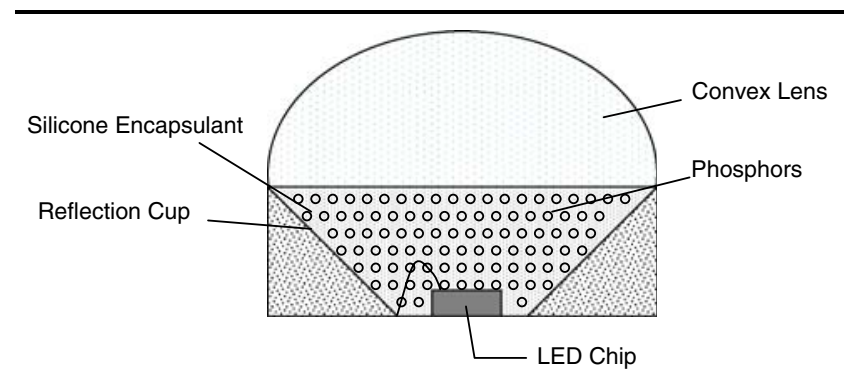

Fig. 1. Schematic cross-sectional view of the dichromatic white LED package. 

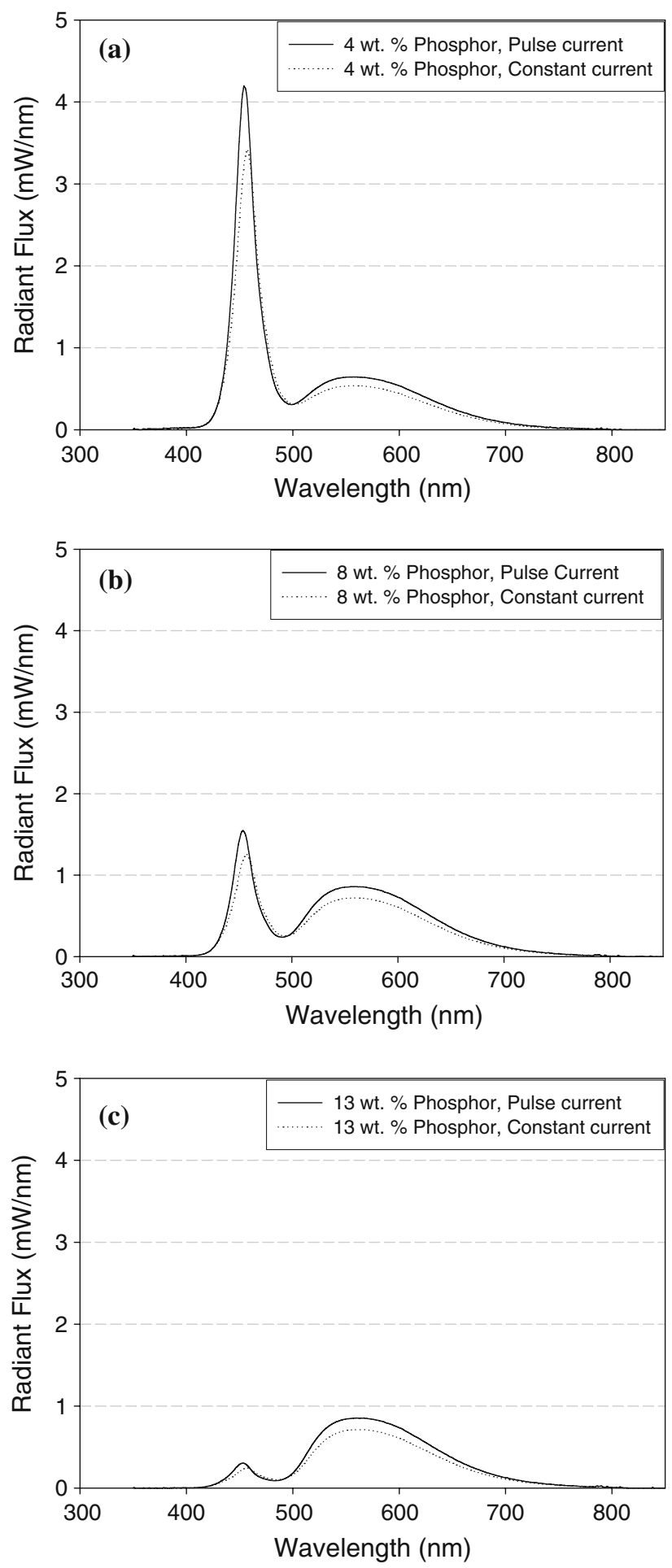

Fig. 2. Emission spectra of LEDs with a phosphor concentration of (a) 4 wt.\%, (b) 8 wt.\%, and (c) 13 wt.\% driven at $350 \mathrm{~mA}$ in pulse and constant current modes.

shows the relative radiation power of LEDs, which is the ratio of radiation power of LEDs driven in constant current mode to that of LEDs driven in pulse current mode at different injection current. The relative radiation power decreases with the increase of injection current and phosphor concentration.

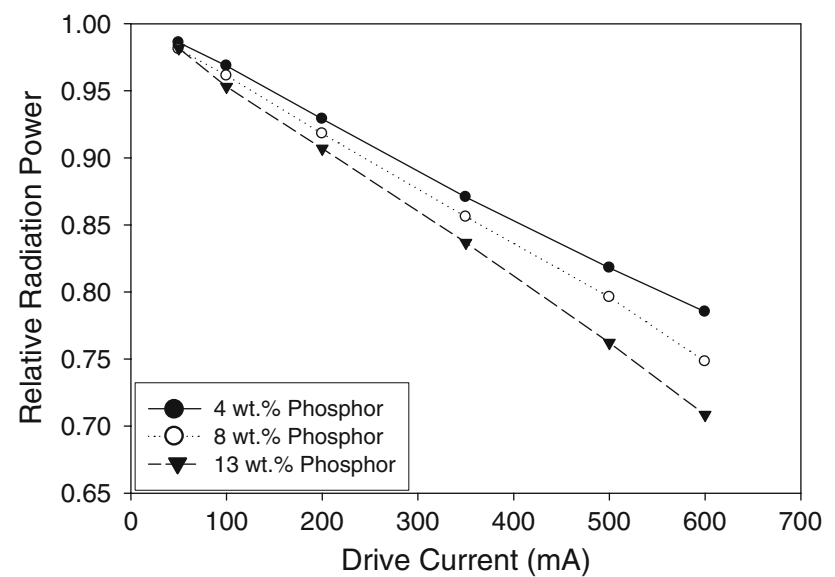

Fig. 3. Relative radiation power of LEDs in constant current mode with respect to the pulse current mode at current levels from $50 \mathrm{~mA}$ to $600 \mathrm{~mA}$.

It should be noted that the lower emission power of LEDs under constant current is due to more heat being generated in the $p-n$ junction. The increased junction temperature leads to lower internal quantum efficiency of the LED and reduction of the phosphor conversion efficiency. ${ }^{4,5}$

The calibration curves of white LEDs with phosphor concentrations of $4 \mathrm{wt} . \%, 8 \mathrm{wt} . \%$, and $13 \mathrm{wt} . \%$, are shown in Fig. 4a-c, respectively. According to the calibration curve, the junction temperatures of white LEDs can be obtained under different drive current. The junction temperatures of LEDs with different phosphor concentrations driven at currents from $350 \mathrm{~mA}$ to $600 \mathrm{~mA}$ are shown in Fig. 5 . The LED with a phosphor concentration of 13 wt.\% has a junction temperature about $10^{\circ} \mathrm{C}$ higher than that of $4 \mathrm{wt} . \%$ driven at a current of $600 \mathrm{~mA}$. As the phosphor concentration increases, a significant portion of light is backscattered by the phosphor and lost within the LED die due to light absorption by packaging materials. This absorbed energy transforms into heat in the die and thus increases the junction temperature of the white LED.

The phosphor conversion efficiency for different phosphor concentrations of white LEDs driven at a current of $350 \mathrm{~mA}$ is shown in Fig. 6. The phosphor conversion efficiency was calculated by dividing the phosphor-converted optical power by the difference of optical power between primary light directly emitted from the LED chip with and without the mix of phosphor. For dichromatic white LEDs using the blue-pumped yellow phosphor, the phosphor conversion efficiencies dropped by around $10 \%$ in both pulse and constant current modes when the phosphor concentration increased from $4 \mathrm{wt} . \%$ to 13 wt.\%; i.e., when more blue light emitted from the LED chip is converted into yellow light with the increase of phosphor material, the phosphor conversion efficiency is lower. The lower conversion efficiency is due to light trapped between phosphor particles and backscattered by phosphor materials. 

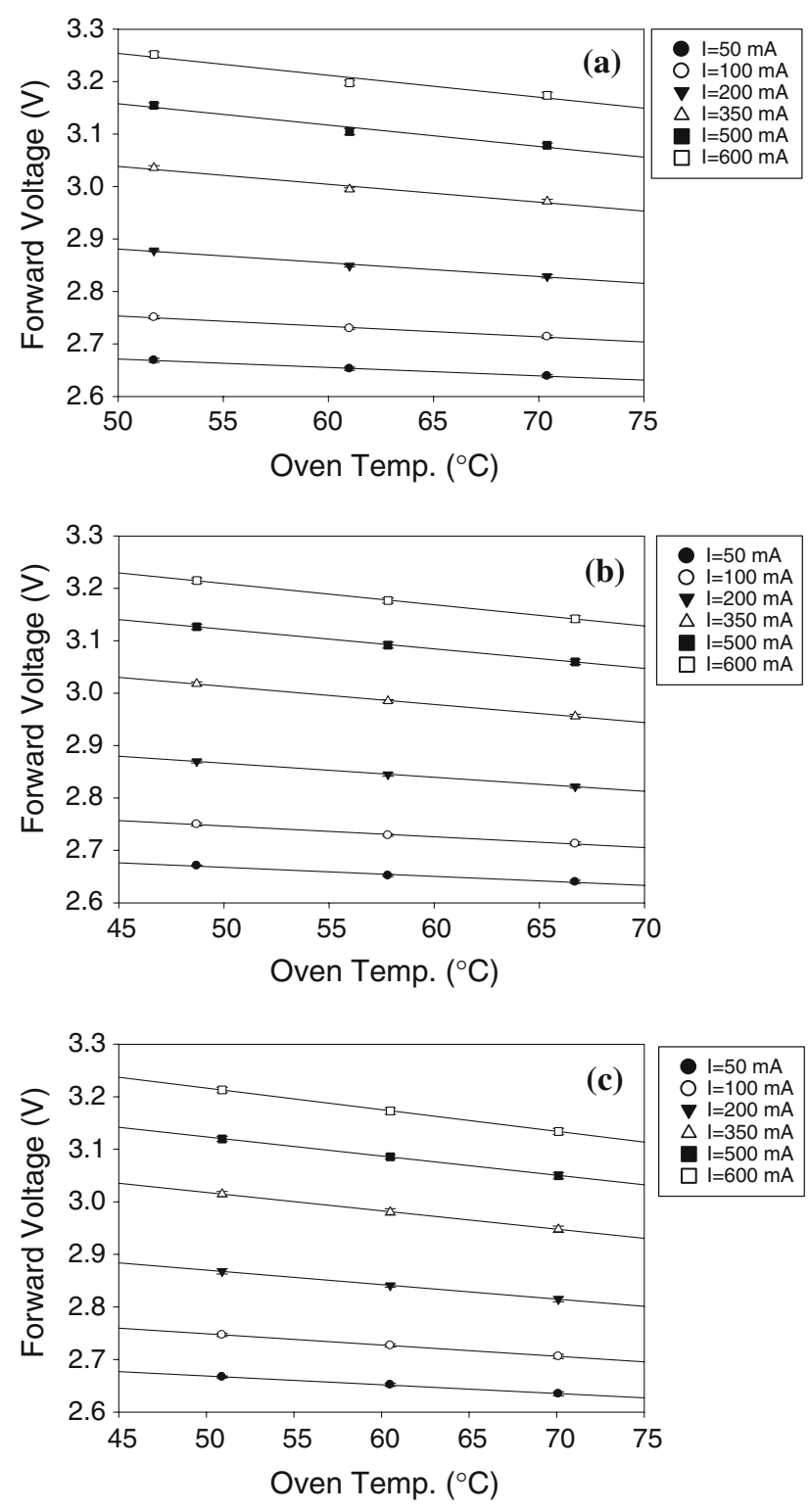

Fig. 4. Calibration curves of LED forward voltage versus oven temperature for LEDs with phosphor concentrations of (a) 4 wt.\%, (b) 8 wt. $\%$, and (c) 13 wt. $\%$ driven at a constant current of $50 \mathrm{~mA}$, $100 \mathrm{~mA}, 200 \mathrm{~mA}, 350 \mathrm{~mA}, 500 \mathrm{~mA}$, and $600 \mathrm{~mA}$, respectively.

As the phosphor concentration increases, more blue light is converted into yellow light and thus lumen increases. On the other hand, the possibility of light trapping and light backscattering also increases. However, as the phosphor concentration increases, there is more blue light being converted to yellow light that has much higher luminous efficacy $(\mathrm{lm} / \mathrm{W})$ than that of blue light. So, there is an optimal lumen output as phosphor concentration increases. This mechanism was also shown by the theoretical simulation of Tran and Shi. ${ }^{10}$

In phosphor-converted white LEDs, optical characteristics depend on the mixing ratio of blue to yellow light, which is related to the parameter of CCT. With the increase of phosphor concentration,

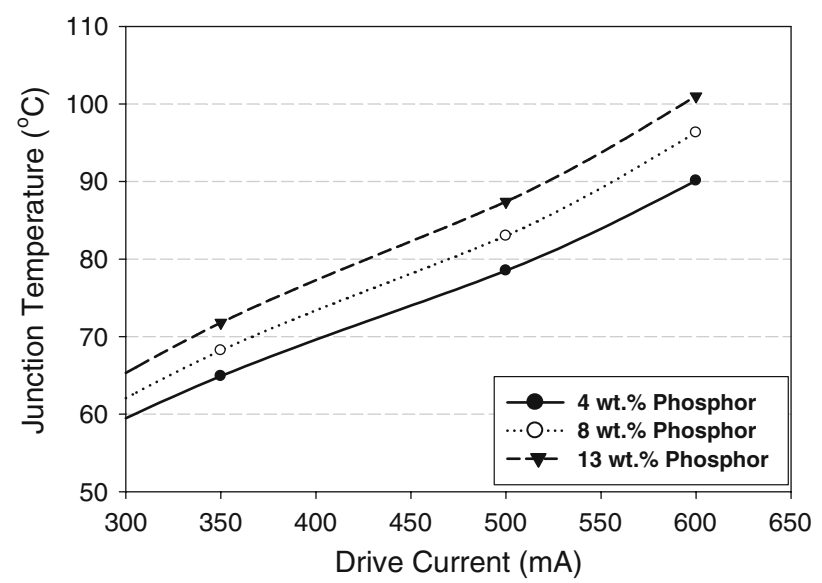

Fig. 5. Junction temperatures of LEDs with different phosphor concentrations driven at currents from $350 \mathrm{~mA}$ to $600 \mathrm{~mA}$ in the same ambient.

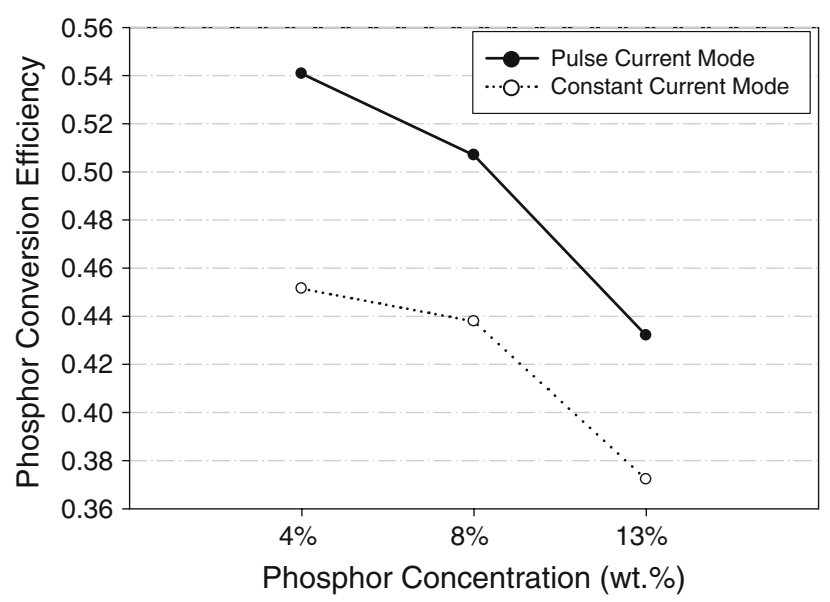

Fig. 6. Phosphor conversion efficiency for dichromatic LEDs driven at $350 \mathrm{~mA}$ with phosphor concentrations of $4 \mathrm{wt} . \%, 8 \mathrm{wt} . \%$, and 13 wt. $\%$, respectively.

more phosphor-converted light is emitted, thus lowering CCT. Figure 7 shows the shifts in CCT with an increase of injection current of LEDs under pulse and constant current. It should be noted that, for the LED with a phosphor concentration of 8 wt.\%, CCT increases with increasing drive current from $50 \mathrm{~mA}$ to $600 \mathrm{~mA}$ and increases faster in the constant current mode compared with the same LED driven in pulse current mode. However, for the LED with a phosphor concentration of $13 \mathrm{wt} . \%$, the CCT only slightly increases with increasing drive current in both pulse and constant current modes. These phenomena are due to the absorption saturation of blue light by phosphor materials and can be explained by the shift in the ratio of blue to yellow light. The intensity ratio of blue light to yellow light, $I_{\mathrm{B}} / I_{\mathrm{Y}}$, can be expressed $\mathrm{as}^{11}$

$$
\frac{I_{\mathrm{B}}}{I_{\mathrm{Y}}}=\frac{1}{\eta S} \frac{1}{\mathrm{e}^{2 \alpha_{\mathrm{B}}\left(I_{\mathrm{B} 0}\right) x}-1} \mathrm{e}^{2 \alpha_{\mathrm{Y}} x},
$$




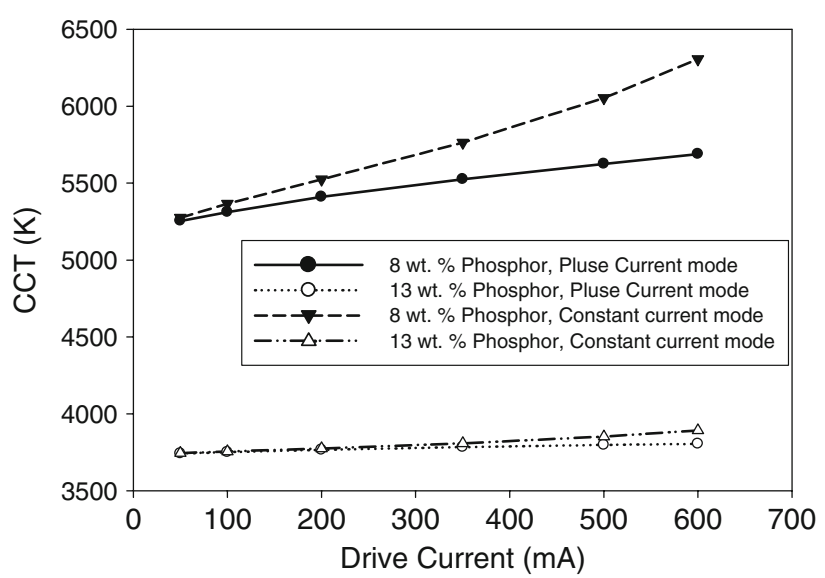

Fig. 7. CCT of LEDs with a phosphor concentration of 8 wt.\% and $13 \mathrm{wt} . \%$ in pulse and constant current modes at different drive currents.

where $I_{\mathrm{B} 0}$ is the initial intensity without the presence of phosphor material, $\alpha_{\mathrm{B}}$ and $\alpha_{\mathrm{Y}}$ are the absorption coefficient of phosphor mixture for the photon energy of blue and yellow light, $x$ is the width of the phosphor mixture layer, $\eta$ is the quantum efficiency of phosphor, and $S$ stands for the Stokes shift.

$I_{\mathrm{B} 0}$ in the constant current mode is always smaller than that in the pulse current mode due to excess heat generated, which increases the possibility of nonradiative recombination. Moreover, at a phosphor concentration of 8 wt.\%, CCT increases with drive current in constant current mode. The reduced phosphor conversion efficiency is due to the increased junction heat and thus leads to an increased ratio of $I_{\mathrm{B}} / I_{\mathrm{Y}}$. With an increase in phosphor concentration, a competing mechanism between reduced phosphor conversion efficiency and increased absorption coefficient is proposed. For a phosphor concentration of 13 wt.\% of white LED, the increase in the absorption coefficient is predominant. The ratio of $I_{\mathrm{B}} / I_{\mathrm{Y}}$ is relatively stable with constant drive current. Therefore, the CCT of white LEDs only slightly increases at high constant current operation.

In white LEDs, light stability also depends on the chromaticity coordinate shift under current regulation, since $I_{\mathrm{B} 0}$ is a direct function of the LED current. A constant color mixing ratio of white LEDs is preferred. The chromaticity coordinate shift is defined as the distance between the old and new coordinates in an $x-y$ chromaticity plot. The chromaticity coordinate shift of LEDs with a phosphor concentration of $8 \mathrm{wt} . \%$ and $13 \mathrm{wt} . \%$ driven at currents from $50 \mathrm{~mA}$ to $600 \mathrm{~mA}$ is shown in Fig. 8. The coordinate shift increases when the phosphor concentration increases from 4 wt.\% to 8 wt.\% and drops drastically when it increases further to $13 \mathrm{wt} . \%$. The increased coordinate shift from a phosphor concentration of $4 \mathrm{wt} . \%$ to that of $8 \mathrm{wt} . \%$ is due to the increased absorption coefficient for blue

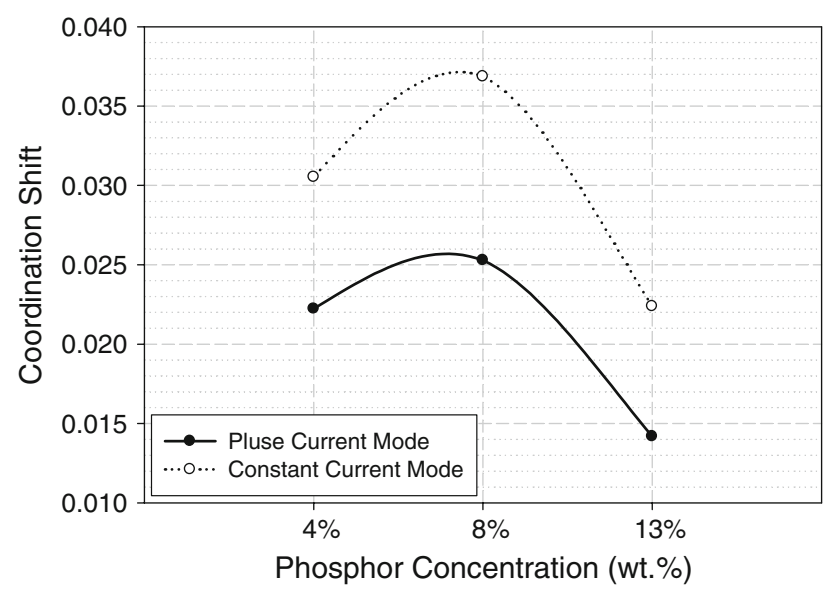

Fig. 8. Chromaticity coordinate shift of LEDs with a phosphor concentration of 8 wt. $\%$ and $13 \mathrm{wt} . \%$ at drive currents from $50 \mathrm{~mA}$ to $600 \mathrm{~mA}$ in pulse and constant current modes.

light. The variation of increased absorption coefficient is relatively large because the absorption coefficient is a function of the intensity of blue light, $I_{\mathrm{B} 0}$. On the other hand, because of absorption saturation in the LED that has a phosphor concentration of $13 \mathrm{wt} . \%$, the dependence of absorption coefficient on $I_{\mathrm{B} 0}$ is small, i.e., reduced coordinate shift. This implies that warm white LEDs have better color stability under current regulation. Moreover, white LEDs under constant current have higher coordinate shifts than under pulse current. The increased coordinate shifts are attributed to the decreased phosphor conversion efficiency, thus increasing the shifts of the ratio of $I_{\mathrm{B}} / I_{\mathrm{Y}}$ from $50 \mathrm{~mA}$ to $600 \mathrm{~mA}$ in constant current operation.

\section{CONCLUSIONS}

In the present work, phosphor-concentrationdependent characteristics of white LEDs driven in different current regulation modes have been investigated. Higher junction temperatures of LEDs under constant current were observed with an increase of phosphor concentration from 4 wt.\% to $13 \mathrm{wt} . \%$. This is due to the increased backscattering and absorption of light within the package. Moreover, the phosphor conversion efficiency in LED packages decreases with the increase of phosphor concentration, whereas LEDs driven under constant current have lower phosphor conversion efficiency than those driven under pulse current. For white LEDs with relatively higher phosphor concentrations, CCT and the chromaticity coordinate shifts are relatively smaller under current regulation in pulse and constant current modes. This can be explained in terms of the increase of saturated absorption coefficient in the relatively higher phosphor concentration of the white LEDs. As the result, high-phosphor-containing white LEDs, i.e., warm white LEDs, have more stable optical characteristics in current regulation applications. 


\section{OPEN ACCESS}

This article is distributed under the terms of the Creative Commons Attribution Noncommercial License which permits any noncommercial use, distribution, and reproduction in any medium, provided the original author(s) and source are credited.

\section{REFERENCES}

1. J. Zhou and W. Yan, Power Electronics Specialists Conference (IEEE PESC, 2007), p. 1436.

2. M.P. Liao, IEEE Photon. Technol. Lett. 19, 2000 (2007). doi:10.1109/LPT.2007.908353.

3. M. Fukuda, Reliability and Degradation of Semiconductor Lasers and LEDs (Boston: Artech House, 1991), p. 39.
4. F. Stern, IEEE J. Quant. Electron. QE-9, 290 (1973). doi:10.1109/JQE.1973.1077478.

5. T. Tamura, T. Setomoto, and T. Taguchi, J. Lumin. 87-89, 1180 (2000). doi:10.1016/S0022-2313(99)00588-8.

6. N. Narendran, Y. Gu, J.P. Freyssinier-Nova, and Y. Zhu, Phys. Status Solidi A 202, 60 (2005). doi:10.1002/ pssa.200510015.

7. M. Dyble, N. Narendran, A. Bierman, and T. Klein, SPIE Proc. 5941, 291 (2005).

8. Y. Gu, N. Narendran, T. Dong, and H. Wu, SPIE Proc. 6337, 63370J1 (2006).

9. Y. Xi, J.Q. Xi, Th. Gessmann, J.M. Shah, J.K. Kim, and E.F Schubert, Appl. Phys. Lett. 86, 031907 (2005). doi:10.1063/ 1.1849838 .

10. N.T. Tran and F.G. Shi, J. Lightwave Technol. 26, 3556 (2008). doi:10.1109/JLT.2008.917087.

11. H. Masui, S. Nakamura, and S.P. Denbaars, Jpn. J. Appl. Phys. 45, L910 (2006). doi:10.1143/JJAP.45.L910. 